\title{
Paracentral acute middle maculopathy and acute macular neuroretinopathy following SARS-CoV-2 infection
}

\author{
Jonathan Virgo $\mathbb{i}^{1} \cdot$ Moin Mohamed ${ }^{2}$
}

Received: 12 June 2020 / Revised: 18 June 2020 / Accepted: 23 June 2020 / Published online: 3 July 2020

(c) The Royal College of Ophthalmologists 2020

\section{To the Editor:}

Ophthalmic complications of COVID-19 are predicted based on prior knowledge of other coronaviruses [1]. Conjunctivitis can be the presenting sign/symptom and its presence correlates with severity [2-4]. Cotton wool spots and retinal microhaemorrhages have also been reported [5]. We report two patients who presented with a new paracentral scotoma following SARS-CoV-2 infection.

Patient 1: A 37-year-old Caucasian female in week 14 of an uncomplicated pregnancy presented with a 1-day history of abrupt onset, faintly colourful, left eye paracentral scotoma. This was 35 days following the onset of a febrile illness with cough and anosmia. SARS-CoV-2 nasopharyngeal swab was not performed during the infection, but subsequently positive serology (IgG) has been confirmed. Past medical history included acephalgic visual migraine aura and right toxoplasma chorioretinitis. Examination showed normal visual acuity, no uveitis and fundoscopy was normal in the left eye. OCT changes correlated with the location of the scotoma (Fig. 1). A focal area of hyper-reflective change in the inner and outer plexiform layers with inner nuclear layer volume loss was seen consistent with paracentral acute middle maculopathy (PAMM). Bloods were normal, including ESR, CRP, lipids, glucose, ANA and anti-phospholipid antibodies. An electrocardiogram and carotid Doppler ultrasound were normal.

Patient 2: A 32-year-old Caucasian male presented with a 4-day history of abrupt onset, faintly colourful, right eye paracentral scotoma. This was 16 days following the onset of nasopharyngeal swab confirmed COVID-19. Past medical history included acephalgic visual migraine aura.

Jonathan Virgo

jdvirgo@nhs.net

Medical Eye Unit, St Thomas' Hospital, London SE1 7EH, UK

2 Medical Retina Department, St Thomas' Hospital, London SE1 7EH, UK
Examination showed normal visual acuity, no uveitis and fundoscopy was normal. Changes on infrared reflectance (white arrow) and OCT correlated with the location of the scotoma (Fig. 2). A focal area of faint outer plexiform layer hyper-reflective change (black arrow) and disruption of the interdigitation zone (white box) were seen consistent with acute macular neuroretinopathy (AMN).

These patients developed PAMM and AMN soon after confirmed SARS-CoV-2 infection and possibly represent postinfectious complications. COVID-19 has been reported in association with acute limb ischaemia, stroke and the so called "paediatric inflammatory multisystem syndrome temporally associated with SARS-CoV-2 infection" [6-8]. PAMM and AMN have similar underlying pathophysiology.

PAMM was first described as a variant of AMN [9], but they are now regarded as distinct conditions with overlapping features. PAMM OCT changes are seen in various retinal vascular diseases, such as retinal vein and artery occlusion. OCT angiography (OCT-A) has provided further support for a retinal vascular aetiology in PAMM and AMN [10-15]. Projection resolved OCT-A distinguishes the intermediate from the deep capillary plexus, which run either side of the inner nuclear layer. Using this technique, it has been shown that PAMM occurs in association with reduced flow in the intermediate, deep and occasionally the superficial capillary plexuses, whereas AMN occurs in association with reduced flow in the deep capillary plexus [15]. Finally, in a series of

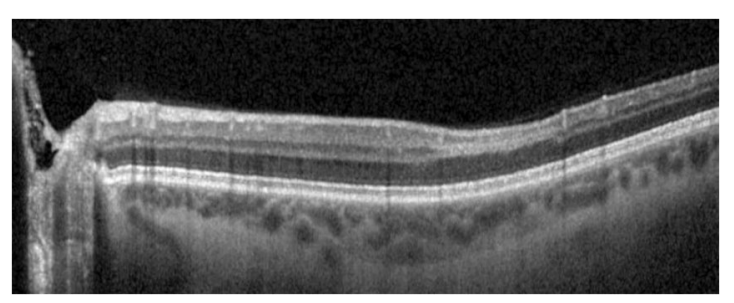

Fig. 1 Optical coherence tomography image from patient 1 . Focal area of hyper-reflective change in the inner and outer plexiform layers with inner nuclear layer volume loss consistent with paracentral acute middle maculopathy. 
Fig. 2 Infrared reflectance and optical coherence tomography images from patient 2. Focal area of IR change (white arrow) due to faint outer plexiform layer hyper-reflective change (black arrow) and disruption of the interdigitation zone (white box) on OCT consistent with acute macular neuroretinopathy.

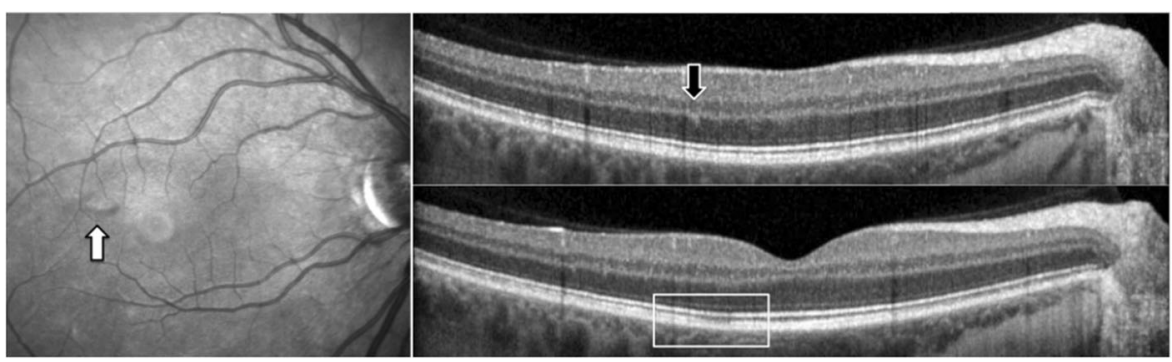

101 AMN cases, an associated infection or febrile illness was reported in $47.5 \%$ [16].

This is the first report of PAMM/AMN following COVID-19. A larger case series is needed to determine if there is a true association.

\section{Compliance with ethical standards}

Conflict of interest The authors declare that they have no conflict of interest.

Publisher's note Springer Nature remains neutral with regard to jurisdictional claims in published maps and institutional affiliations.

\section{References}

1. Seah I, Agrawal R. Can the coronavirus disease 2019 (COVID-19) affect the eyes? a review of coronaviruses and ocular implications in humans and animals. Ocul Immunol Inflamm. 2020;28:391-5.

2. Scalinci SZ, Trovato Battagliola E. Conjunctivitis can be the only presenting sign and symptom of COVID-19. IDCases. 2020;20: e00774.

3. Wu P, Duan F, Luo C, Liu Q, Qu X, Liang L, et al. Characteristics of ocular findings of patients with coronavirus disease 2019 (COVID19) in Hubei Province, China. JAMA Ophthalmol. 2020;138:575-8.

4. Loffredo L, Pacella F, Pacella E, Tiscione G, Oliva A, Violi F. Conjunctivitis and COVID-19: a meta-analysis. J Med Virol. 2020. https://doi.org/10.1002/jmv.25938.

5. Marinho PM, Marcos AAA, Romano AC, Nascimento $H$, Belfort R Jr. Retinal findings in patients with COVID-19. Lancet. 2020;395:1610.

6. Bellosta R, Luzzani L, Natalini G, Pegorer MA, Attisani L, Cossu LG et al. Acute limb ischemia in patients with COVID-19 pneumonia. J Vasc Surg. 2020;S0741-5214:31080-6. https://doi. org/10.1016/j.jvs.2020.04.483.

7. Oxley TJ, Mocco J, Majidi S, Kellner CP, Shoirah H, Singh IP, et al. Large-vessel stroke as a presenting feature of Covid-19 in the young. N. Engl J Med. 2020;382:e60.

8. European Centre for Disease Prevention and Control. Paediatric inflammatory multisystem syndrome and SARS-CoV-2 infection in children. Stockholm: ECDC; 2020.

9. Sarraf D, Rahimy E, Fawzi AA, Sohn E, Barbazetto I, Zacks DN, et al. Paracentral acute middle maculopathy: a new variant of acute macular neuroretinopathy associated with retinal capillary ischemia. JAMA Ophthalmol. 2013;131:1275-87.

10. Pecen PE, Smith AG, Ehlers JP. Optical coherence tomography angiography of acute macular neuroretinopathy and paracentral acute middle maculopathy. JAMA Ophthalmol. 2015;133:1478-80.

11. Rahimy E, Kuehlewein L, Sadda SR, Sarraf D. Paracentral acute middle maculopathy: what we knew then and what we know now. Retina. 2015;35:1921-30.

12. Nemiroff J, Kuehlewein L, Rahimy E, Tsui I, Doshi R, Gaudric A, et al. Assessing deep retinal capillary ischemia in paracentral acute middle maculopathy by optical coherence tomography angiography. Am J Ophthalmol. 2016;162:132.

13. Kulikov AN, Maltsev DS, Leongardt TA. Retinal microvasculature alteration in paracentral acute middle maculopathy and acute macular neuroretinopathy: a quantitative optical coherence tomography angiography study. Retina Cases Brief Rep. 2018. https:// doi.org/10.1097/ICB.0000000000000709.

14. Chu S, Nesper PL, Soetikno BT, Bakri SJ, Fawzi AA. Projectionresolved OCT angiography of microvascular changes in paracentral acute middle maculopathy and acute macular neuroretinopathy. Investig Ophthalmol Vis Sci. 2018;59:2913-22.

15. Chen YC, Chen SN. Microvascular change in acute macular neuroretinopathy by using optical coherence tomography angiography. Taiwan J Ophthalmol. 2019;9:118-21.

16. Bhavsar KV, Lin S, Rahimy E, Joseph A, Freund KB, Sarraf D, et al. Acute macular neuroretinopathy: a comprehensive review of the literature. Surv Ophthalmol. 2016;61:538-65. 